\title{
NEW FMRI PARADIGM TO STUDY BEHAVIORAL AND NEURAL BASES OF VISUAL WORKING MEMORY
}

\author{
A.R.Dores ${ }^{1,2}$, F.Barbosa ${ }^{3}$, I.Almeida ${ }^{4}$, S.Guerreiro ${ }^{3,4}$, B.M.Rocha ${ }^{5}$, I.P.Carvalho ${ }^{5}$, A.J.Marques ${ }^{2}$, \\ L.D.Sousa ${ }^{1}$, A.Castro-Caldas ${ }^{6,7}$
}

${ }^{1}$ Instituto de Ciências Biomédicas Abel Salazar, Universidade do Porto, 'Laboratório de Reabilitação Psicossocial, Faculdade de Psicologia e de Ciências da Educação da Universidade do Porto e Escola Superior de Tecnologia da Saúde do Instituto Politécnico do Porto, ${ }^{3}$ Faculdade de Psicologia e de Ciências da Educação da Universidade do Porto, ${ }^{4}$ Centro de Reabilitação Profissional de Gaia, ${ }^{5}$ Faculdade de Medicina da Universidade do Porto, Porto, ${ }^{6}$ Brain Imaging Network Portugal (BING/ANIFC), ${ }^{7}$ Instituto de Ciências da Saúde, Universidade Católica Portuguesa, Lisbon, Portugal

Introduction: Working memory (WM) is a cognitive process that refers to storing information in a temporary system that allows monitoring and handling this information.

Objectives: To propose and validate a new fMRI paradigm to study WM and its neuroanatomical substrates, using a language-free adaptation of the 2-back working memory task in order to avoid cultural and educational bias.

Aims: To test the hypothesis that the proposed paradigm would produce an increase of the BOLD signal in specialized areas for spatial WM (Superior Frontal Sulcus) and areas for monitoring and handling this information (Dorsolateral Prefrontal Cortex) in healthy participants. We also expected a significantly higher behavioral performance in this group than in subjects with suspected WM impairment due to acquired brain injury.

Methods: 10 neurologically healthy participants and $11 \mathrm{ABI}$ participants performed the task in a block design experiment with four runs. These observed a 9-square matrix with one of the squares painted black and pressed a button each time the black square was the same as the one two trials back. We analyzed behavioral performance and brain activation in repeated trials.

Results: Higher BOLD activation of brain regions was associated with the performance of the WM task in the healthy group, as well as better behavioral performance. We also present behavior results of both groups, so these data can be compared to other clinical groups with suspected WM deficits.

Conclusions: This task may be used as a research methodology for behavioral and neuroimaging studies of visual WM in block-design paradigms. 